American Journal of Applied Sciences 7 (3): 290-300, 2010

ISSN 1546-9239

(C) 2010Science Publications

\title{
Ultrasonic Dental Scaler Performance Assessment with an Innovative Cavitometer
}

\author{
${ }^{1}$ Antonio Henrique Alves Pereira, ${ }^{2}$ Camila Tirapelli and ${ }^{1}$ Lucimar Antonio Rodolpho \\ ${ }^{1}$ Division of Research and Development, ATCP Physical Engineering, \\ Rua Monteiro Lobato, 1601, 13569-290, Sao Carlos, SP, Brazil \\ ${ }^{2}$ Department of Dental Materials and Prosthesis, School of Dentistry of Ribeirao Preto, \\ University of Sao Paulo, Avenida do Cafe, s/n, 14041904, Ribeirao Preto-SP, Brazil
}

\begin{abstract}
Problem statement: There is no specific instrument to test ultrasonic dental scalers used in dentistry. Approach: The aim of this research was to develop and test an innovative cavitometer for quantitative and automatic performance assessments of Ultrasonic Dental Scalers (UDS) through the acoustic emission produced by cavitation induced by the tip of the UDS ultrasonic transducer when immersed in aqueous solution. Firstly, an optimized acoustic emission sensor specifically for UDS ultrasonic transducers, software to process the acoustic emission sensor signal and to calculate a Figure of Merit (FM) and a hydraulic-pneumatic circuit were developed; these parts together constituted the cavitometer. To validate the developed cavitometer, two groups of UDS transducers were tested with the cavitometer and with one cleaning test; 48 units of the Jet1 UDS model and 12 units of the Jet2 model (Gnatus, Ribeirao Preto, Brazil) were tested. Sixteen assessments were carried out with each transducer, for a total of 768 measurements with Jet1 and 192 with Jet2. Spearman test was employed to observe correlations between the measurements from the different methods. Jet1 transducers were compared with Jet2 employing Student $t$ test for cavitometer assessments and Mann Whitney $U$ test for the cleaning test assessments. Results: Was found correlation between the cavitometer and cleaning results. The developed cavitometer detected a significant difference between average results for the two transducer groups (Jet1: $4.3 \pm 0.7$ Vs Jet2: $6.2 \pm 0.5$ ) and this was also observed for the cleaning test (Jet1: $7.0 \pm 0.8$ Vs Jet2: $8.7 \pm 0.4$ ) Conclusion: The results showed that the developed cavitometer is adequate for the UDS quantitative performance assessment, particularly for replacement of the cleaning test for industrial quality control.
\end{abstract}

Key words: Quality control, equipments in dentistry, ultrasonic dental scalers, cavitometer, cavitation

\section{INTRODUCTION}

The removal of dental plaque and calculus by ultrasonic devices was first evaluated in the 1950s (Johnson and Wilson, 1957). Currently, it is a widely accepted clinical procedure and probably the most commonly recognized application of power ultrasonic in dentistry, followed by ultrasonic cleaning baths. The effects of removing dental plaque and calculus with ultrasonic devices have been extensively studied (Zitterbart, 1987; Guentsch and Preshaw, 2008; Walmsley et al., 2008) and in this context, the removal efficiency is attributed to the chipping action of the probe tip and cavitation activity induced in the ultrasonic transducer cooling water (Walmsley et al., 1984; Drisko, 1998; Oda et al., 2000; Arabaci et al., 2007). Ultrasonic Dental Scalers (UDS) are driven by a magnetostrictive or piezoelectric transducer that converts electrical energy into mechanical vibrations at resonance frequencies that are usually between 25 and $45 \mathrm{kHz}$. The differences between magnetostrictive and piezoelectric UDS transducers (Busslinger et al., 2001) and the different tips used (Lea et al., 2003a; 2003b; 2004; 2006) have also been studied.

Despite its wide use and great importance to dentists (Clark, 1969), there is not a recommended standard method (e.g., ASTM or ISO) to assess UDS performance. However, some important progress has been achieved to develop and establish methods to assess UDS performance. The focus has primarily been in two directions: Measuring the tip vibration amplitude and measuring the cavitation intensity induced by the tip. To measure the vibration amplitude, optical microscopes have been employed (Walmsley et al., 1986) and, more recently, so have laser Doppler vibrometers (Lea et al., 2002; 2004; 2009a; 2009b;

Corresponding Author: Antonio Henrique Alves Pereira, ATCP Physical Engineering, Rua Monteiro Lobato, 1601, 13569-290, Sao Carlos, SP, Brazil Tel/Fax: +55 1633077899 
Felver et al., 2009). To measure the cavitation, methods based on sonochemical effects have been employed (Walmsley et al., 1986; Lea et al., 2005) and methods based on quantifying the cavitation acoustic emission induced by the tip submerged in an aqueous solution have also been proposed (Felver et al., 2009; Zeqiri et al., 2003).

The methods based on measuring the tip amplitude of vibration are conceptually straightforward but require an experienced operator and, in some cases, expensive equipment, such as laser Doppler vibrometers. The sonochemical method, as designed by Lea et al. (2005), has great potential to become practical; however, it demands modifications in the UDS transducer to avoid diluting the cavitation sensitive chemical solution with the cooling water. The sonochemical method, as designed by Walmsley et al. (1986), is also practical; however, it requires filling the whole UDS hydraulic circuit with a substance that may decrease the UDS biosafety, which causes the method to fail industry quality control. In these methods, the sonochemical effect can be measured by the optical density variation of a chemical solution or with a spectrometer. Therefore, these encouraging prospects for UDS performance assessment, the sonochemical and amplitude of vibration methods, have critical drawbacks for serial quality control in industry such as the need for experienced operator, the demand of supplies, the use of chemicals in the UDS hydraulic circuit and, in some cases, high cost and complex equipment requirements.

The detection and quantification of cavitation induced by the UDS tip in an aqueous solution was investigated with acoustic emission and sonochemical (luminol) methods and correlated with the amplitude of vibration by Felver et al. (2009). The results from these authors indicated that a system able to measure the components of the acoustic emission associated with inertial or transient cavitation could be as effective as the vibration amplitude and sonochemical methods to UDS performance assessment. Considering that the cavitation depends on the amplitude of vibration and that both the amplitude of vibration and cavitation play a key role in UDS performance (Walmsley et al., 1984; Drisko, 1998; Oda et al., 2000; Arabaci et al., 2007), one can expect to find a direct correlation between the UDS performance and cavitation occurrence; however, care must be taken because the cavitation occurrence is susceptible to several parameters, such as tip type and aqueous solution, that are not necessarily directly correlated with the UDS performance. The information that can be extracted from the cavitation acoustic emission is already used for performance assessment of ultrasonic cleaning systems (Leighton, 2007), there are commercial cavitometers for this purpose as well as standardization initiatives (Hodnett and Zeqiri, 1997). However, until now, the cavitometers described in the literature were mainly projected with sensors to be immersed in a liquid medium subjected to ultrasound and the difference between an ultrasonic cleaning tank and a UDS ultrasonic transducer makes the design of an immersible acoustic emission sensor non-optimal for practical and reproducible UDS performance assessment. In the UDS transducer, the cavitation occurs around the tip and not in a large liquid surrounding, consequently the tip positioning regarding the sensor becomes a critical parameter to ensure the test reproducibility.

Due to widespread UDS use in dentistry and to the restrictions of the current techniques for performance assessment, there is a need for an automated quantitative solution for industrial UDS quality control able to substitute test procedures based on human judgment. Considering this fact, this research develops an acoustic emission sensor with an innovative design optimum for UDS transducers. The working principle of the cavitometer described here is based in the measurement of the acoustic emission noise produced by cavitation induced by the tip of the ultrasonic transducer when immersed in aqueous solution. Software was also developed to process automatically the acoustic emission sensor signal based on the method proposed by Frohly et al. (2000) and a hydraulic circuit to test the UDS transducer internal hydraulic seal. Those parts constitute the cavitometer.

The industrial version of the cavitometer does not demand an experienced operator and its estimated cost is 12,500 USD including four cavitometer sensors, a computer equipped with an acquisition board and processing software. It is able to automatically test 04 UDS at the same time and to give a deterministic result (approved or not approved) without human judgment. The typical testing time for 04 UDS is about half an hour, such that the cavitometer is able to test 64 UDS in a day with 8 work hours. The cavitometer laboratory version for scientific experiments, without a computer, acquisition board, or software-only the sensor-is estimated to cost 1,500 USD. To use this version, it is necessary to have a signal acquiring apparatus (a digital oscilloscope for instance) and post-processing software available. Both the industrial and laboratorial versions allow direct observation of the tip and possibly the realization of other tests simultaneously, such as luminescence tests; experiments are being designed to explore this possibility. 


\section{MATERIALS AND METHODS}

The cavitometer development:

Acoustic emission sensor: The main element of the cavitometer developed is the acoustic emission sensor illustrated in Fig. 1. It is a cylindrical set with an optimized design for UDS transducers with reproducible positioning and loading. It was projected to detect acoustic emissions with a flat response up to $50 \mathrm{kHz}$. The sensor chamber (Fig. 1, S02 element) has a closed end and a duct System (S08) to allow the cooling water to flow and, at the same time, to ensure a constant fill level (S09). The sensor itself is a small tank where the transducer tip (S03) is submerged and subjected to a constant acoustic load. The chamber's aqueous solution content is continuously renewed by the transducer cooling flow promoted by the hydraulic-pneumatic circuit at constant pressure. The acoustic emissions are captured and converted into an electrical signal with a piezoelectric disk (S05) of lead zirconate titanate (Noliac, Czech Republic) as the sensitive element; this piezoelectric element was bonded in the cylinder closed end and a coaxial cable (S07) connects it to the computer acquisition card NI 6220 (National Instruments, USA).

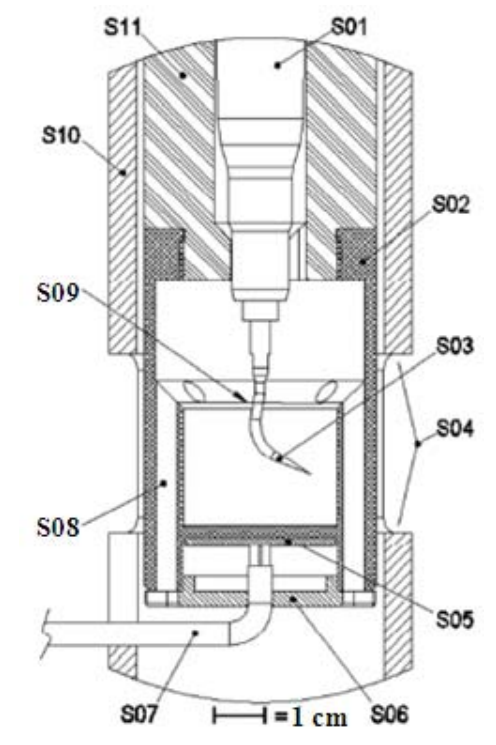

Fig. 1: (A) Cross section of acoustic emission sensor. Main parts: UDS transducer (S01), sensor chamber (S02), transducer tip (S03), casing windows (S04), piezoelectric disk (S05), bottom cover (S06), coaxial cable (S07), duct system (S08), fill level (S09), casing (S10) and transducer support (S11). (B) The cavitometer laboratory version for scientific experiments
The sensor chamber is coupled to a support (S11), which ensures the longitudinal positioning reproducibility of the UDS transducer; this support is matched to the UDS transducer shape, which differs from one manufacturer to another the sensor has circular symmetry, which reduces the influence of the UDS transducer's angular position on the reproducibility. The sensor chamber (S02) was built with a transparent polymer to permit visual verification of the tip position, the aqueous solution level and eventual turbulence or the occurrence of bubbles. Around the sensor chamber there is a casing (S10), which collects and returns the running aqueous solution to the hydraulic circuit auxiliary reservoir; this casing has windows (S04) that permit the sensor chamber and its contents to be observed.

Standard aqueous solution: The use of a standard aqueous solution is crucial to the intensity reproducibility of the cavitation induced by the transducer tip. The presence and sizes of nucleation sites, viscosity, surface tension and temperature are determinant to the cavitation threshold and intensity for a given ultrasonic intensity level (Atchley et al., 1988). In this study, an aqueous solution of filtered $(1 \mu \mathrm{m})$ tap water with the addition of $3 \%$ in weight of biodegradable low toxicity tensoactive detergent (active component: Linear alkyl-benzene sodium sulfonate) that does not compromise the UDS biosafety was used. The addition of the tensoactive detergent contributes to the aqueous solution stabilization and reduces the surface tension inhibiting the formation of gas bubbles in the sensor chamber walls. The occurrence of bubbles can easily attenuate the acoustic emission sensor sensitivity in excess of- $12 \mathrm{dBV}$. The temperature of the aqueous solution was not controlled.

Hydraulic-pneumatic circuit: The hydraulicpneumatic circuit was developed with two main objectives: Test the ultrasonic transducer's internal sealing without causing turbulence inside the sensor chamber and permit the use of a standard aqueous solution saving water.

It is important to fix the pressure of the aqueous solution supplied to the UDS during the tests because it determines the flow rate inside the acoustic sensor chamber, which can decrease its sensitivity when turbulence occurs with the same attenuation as the gas bubbles. Testing the internal hydraulic sealing of the transducer at working conditions is important because a seal failure allows water infiltration in the transducer piezoelectric ceramic location, which causes damage. In the case of magnetostrictive transducers, the infiltration 
problem does not occur due its different scheme. The pressure was set to the highest value possible to increase the seal test efficiency without running the risk of causing turbulence inside the sensor chamber; this value was found to be two Bar. To check if infiltration occurred, the UDS transducer Direct Current (DC) electrical resistance is measured after the test and must be higher than $10^{6}$ ohms $(1 \mathrm{M} \Omega)$.

As a closed loop, the hydraulic-pneumatic circuit saves water. To be fully filled it demands $15 \mathrm{~L}$ of water and $0.45 \mathrm{~kg}$ of detergent, which must be renewed every week. Otherwise, $320 \mathrm{~L}$ of water and $9.6 \mathrm{~kg}$ of detergent would be necessary per week considering 64 UDS tests per workday consuming $1 \mathrm{~L}$ of aqueous solution each.

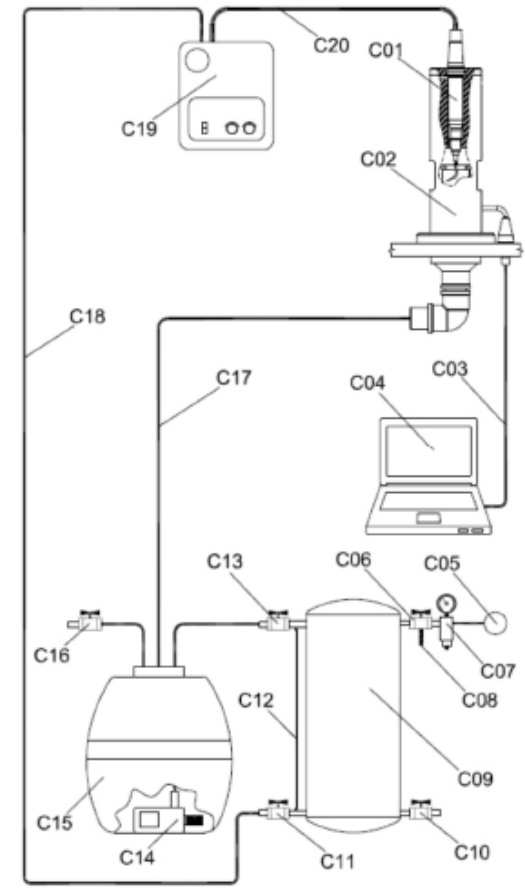

Fig. 2: Schematic plan of the hydraulic-pneumatic circuit. Main parts: UDS transducer (C01), acoustic emission sensor ( $\mathrm{C} 02)$, connation to the acquisition card ( $\mathrm{C} 03)$, computer (C04), dry and filtered compressed air line (C05), 3/2-way valve (C06), pressure regulator (C07), depressurization way (C08), pressurization cylinder (C09), discharge valve (C10), UDS feeding valve (C11), level indicator (C12), refill valve (C13), refill ump (C14), auxiliary tank (C15), water inlet valve (C16), aqueous solution return (C17), UDS feeding pipe (C18), UDS electronics (C19) and transducer electrical and hydraulic connection (C20)
Figure 2 shows the hydraulic-pneumatic circuit and its main elements: The pressurization Cylinder (C09), the pressure regulator $(\mathrm{C} 07)$, the auxiliary tank $(\mathrm{C} 15)$ and the refill pump (C14). To pressurize the Cylinder (C09), the pressure regulator is connected to a dry and filtered compressed air line (C05). The pressure regulator is positioned in series with the 3/2-way valve (C06), which has its third way open to the atmosphere (C08) to permit the cylinder depressurization to refill. After the aqueous solution flows through the UDS transducer (C01) and the acoustic emission sensor $(\mathrm{C} 02)$, it returns to the auxiliary tank (C15), where it remains during the full discharge time of the pressurization cylinder (C09).

After the complete pressurization cylinder discharge, which is monitored by the indicator level (C12), it is necessary to return the solution that should now be completely stored in the auxiliary tank (C15). After use, the aqueous solution should rest in the auxiliary reservoir or in the pressurization cylinder at atmospheric pressure to avoid gasification.

It is important to note that the UDS studied here is composed of three different parts, the electronic/hardware (or generator), the piezoelectric transducer and a tip.

Signal processing and figure of merit: The signal processing of the acoustic emission sensor is performed by software programmed in Labview ${ }^{\circledR}$ based on the method proposed by Frohly et al. (2000) and improved by Liang et al. (2006), where a "Cavitation Noise Power indicator" (CNP) is calculated by integrating the noise power spectrum generated by the shock wave accompanying the bubble collapses neglecting the contribution of the fundamental frequency, harmonics and sub-harmonics. Frohly et al. (2000) investigated the correlation between the white noise and cavitation activity considering a spectrum range from $0-5 \mathrm{MHz}$ using a transducer with an operational frequency at $1 \mathrm{MHz}$; Liang et al. (2006) considered the spectrum range from $0-500 \mathrm{kHz}$ using a transducer with an operational frequency around $26 \mathrm{kHz}$. In this research, the spectrum range considered was from $5-17.5 \mathrm{kHz}$ and the transducer operation frequency was around 29 $\mathrm{kHz}$. This relatively low frequency spectrum range, in contrast with the mentioned references, was chosen because it was shown to be efficient in pilot tests to maximize the correlation between the developed cavitometer assessment results and the cleaning test and because this range eliminates the demand for a broadband acoustic sensor and allows the use of cost effective acquisition boards with low sample rates: 
$A_{d B V}(f)=20 \log \left[A_{V}(f)\right]$

Figure 3 shows the fast Fourier transform of the acoustic sensor signal characterizing a UDS at the power level extremes with five points per window adjacent-averaging smoothing.

The dashed region indicates the frequency range of the integration $(5-17.5 \mathrm{kHz})$ and clearly shows the noise level increasing with the power level change from 20$100 \%$. It is also possible to observe the fundamental frequency and the first odd harmonic lines in 28.5 and $57 \mathrm{kHz}$. The power spectrum region around $80-85 \mathrm{kHz}$ is probably distorted by the planar resonance frequency of the acoustic sensor active element, a piezoelectric disc $30 \mathrm{~mm}$ in diameter and $2 \mathrm{~mm}$ thick and a planar resonance frequency situated between 70 and $85 \mathrm{kHz}$. The signal acquisition of this example was performed at $250 \mathrm{kS} \mathrm{sec}{ }^{-1}$ with 16 bits and 1024 points. The power spectrum is in $\mathrm{dBV}$ and calculated from the linear amplitude as specified in Eq. 1.

$\mathrm{CNP}=\left|\left(\int_{5 \mathrm{kHz}}^{17.5 \mathrm{kHz}} \mathrm{A}_{\mathrm{dBV}}(\mathrm{f}) \mathrm{df}\right)^{-1}\right|$

The CNP is calculated by integrating the power spectrum in logarithmic scale (dBV) to enhance the noise contribution as proposed by Frohly et al. (2000) and by neglecting the contribution of lines in agreement with the improvement proposed by Liang et al. (2006). A line was expected to be found around $14-15 \mathrm{kHz}$ corresponding to the $\mathrm{f} / 2$ sub-harmonic that is not a significant parameter of cavitation intensity (Frohly et al., 2000 ) and should be ignored; however, it was not observed at any power level for the UDS tested and aqueous solution used. As the absolute value of the spectrum integration decreases with increasing power due to amplitude calculation in $\mathrm{dBV}$, which brings always values smaller or is equal to zero (the acquisition board full scale is 1 Volt), the CNP was calculated as the inverse of the absolute value of the integral shown in Eq. 2.

$\mathrm{FM}=\alpha+\beta(\mathrm{CNP})$

To make the developed cavitometer friendlier to a non-research operator at the industrial production line quality control, a scale change was applied to convert the CNP to a figure of merit, FM, with values between 0 and 10 for the typical UDS performance range. This scale change uses Eq. 3, where $\alpha$ and $\beta$ are arbitrary constants adjusted at $2 \times 107$ and 33 , respectively.

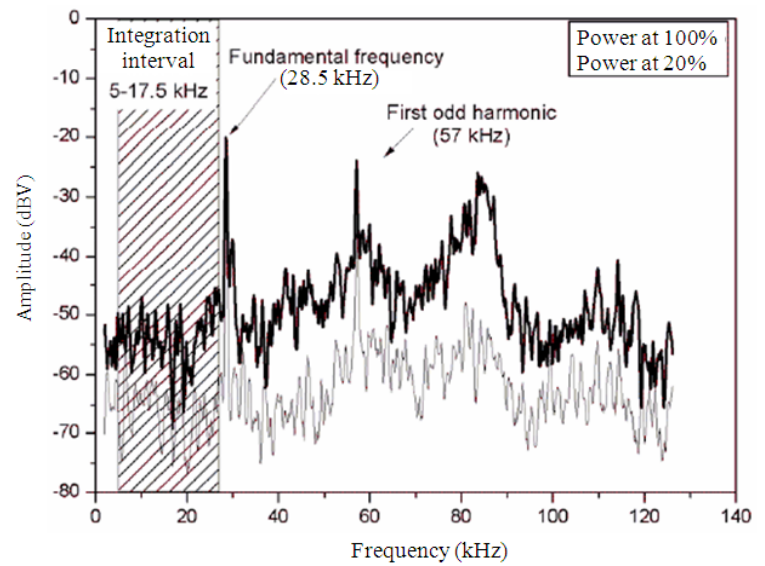

Fig. 3: Fast Fourier transform of the acoustic sensor signal characterizing a UDS transducer at the UDS power level extremes. The dashed region indicates the frequency range of integration (5$17.5 \mathrm{kHz}$ ) and clearly shows the noise level increase due to $20-100 \%$ power level change. It is also possible to observe the fundamental frequency and first odd harmonic lines in 28.5 and $57 \mathrm{kHz}$

The FM and the CNP contain the same information and both are a figure of merit in the strict sense; it is also important to emphasize that they do not correspond to a physical quantity.

$\mathrm{mFM}=\frac{1}{\mathrm{i}} \sum_{\mathrm{i}} \mathrm{FM}(\mathrm{i})$

After the programmed testing time, the software must calculate and output the average $\mathrm{FM}(\mathrm{mFM})$ for the interval of time as indicated in Eq. 4, where the parameter $i$ is equal to the total number of acquisitions. The FM is the instantaneous figure of merit value at a determined time, while the $\mathrm{mFM}$ is the average of the total (equal to i) FM instantaneous values measured along the whole test.

\section{Cavitometer validation:}

Acoustic emission sensor frequency band: To verify the linear frequency range of the acoustic emission sensor we used impedance spectroscopy. The impedance module $(|Z|)$ as a function of frequency was measured using an ultrasonic transducer analyzer, TRZ03 (ATCP Physical Engineering, São Carlos, Brazil), starting from $1 \mathrm{kHz}$ to detect the first resonance frequency. 


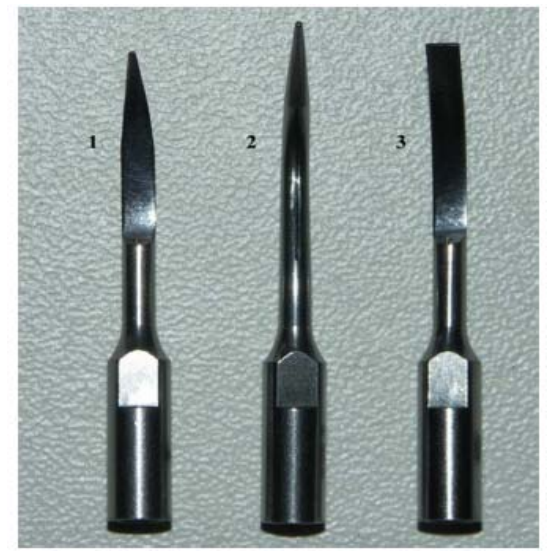

Fig. 4: Illustration of the tip types. Tip type 2 was selected to perform the cavitometer validation

Cavitometer test conditions: The cavitometer test conditions were defined such that the software must perform the sensor signal acquisition, calculate the FM and store the result every six seconds for $5 \mathrm{~min}$. After the programmed testing time, the software must calculate and output the average FM (mFM) from the five-minute interval. The pressurization cylinder pressure must be set to two Bar.

The longitudinal transducer tip position inside the chamber is always the same and determined with a high degree of reproducibility by the flawless matched transducer support (S11 in Fig. 1). Regarding the angular positioning, the acoustic sensor set has cylindrical symmetry, which minimizes the angular position influence; in fact, the angular position is the sole UDS transducer positioning degree of freedom. As a precaution, the angular tip position inside the chamber was also fixed to avoid any slight influence on the sensor's sensitivity; it was defined to locate the tip with its extremity aligned with the middle of the case window (S04 in Fig. 1). This point was marked to assure the correct positioning.

How the type and the UDS power settings affect the mFM values were investigated to decide the tip type and power level to be used. Five ultrasonic transducers used in the UDS model Jetl were assessed three times each on the same UDS hardware/electronics with the following power settings: 20, 40, 60, 80 and 100\%. Later, the same five UDS Jet1 transducers were assessed with the three different tip types (Fig. 4) $(n=5$ for each tip type) on the same UDS hardware/electronics, with the power set at $80 \%$.

Through these preliminary assessments, tip type 2 was defined and the power level was set to $80 \%$ for the standard test conditions. Tip type 2 was chosen because it was found to be less prone to be broken by fatigue and to be corroded by the cavitation, in comparison to types 1 and 3 in the case of use the same tip for several different UDS tests in sequence, probably due to its round shape face compared to the others flat shape. The power level was set to $80 \%$ because it is in the power region where the FM is more sensitive to power level changes.

Cavitometer assessments: To validate the developed cavitometer, a large number of assessments were outlined and executed to verify the coherence of the $\mathrm{mFM}$ values face to the cleaning test and to compare the Jet1 and Jet2 performance. Forty-eight ultrasonic transducers used in the UDS model Jet1, 12 ultrasonic transducers used in UDS model Jet2 and 4 UDS Jet hardware/electronics units (Gnatus, Ribeirão Preto, Brazil) were loaned randomly. All tips, hardware/electronic UDS and transducers were new. The UDS were tested by the cavitometer at the defined conditions (pressure, tip type, positioning and power level) with a programmed testing time of five minutes. Four repeated assessments were performed in each one of the four hardware/electronics permutations with each of the 48 Jet 1 and of the 12 Jet2 UDS transducers; i.e., each transducer was measured 16 times. Thus, $768 \mathrm{mFM}$ values were recorded with the Jet1 model and $192 \mathrm{mFM}$ values with the Jet 2 model.

Cleaning test method: The cleaning test is the qualitative method to be replaced by the developed cavitometer; it measures the UDS ability to remove epoxy paint from a metal plate. It is effective to a certain extent, but is dependent on an experienced operator with self discipline to ensure the test confidence. These test characteristics are undesirable for an industrial environment, particularly when it is necessary more than one operator and in holiday periods. Beyond that, the continuous and repetitive movements to remove the paint may cause Repetitive Strain Injury (RSI) in the operator. These drawbacks of the cleaning test were the motivation for the cavitometer development.

As the comparative method for cavitometer validation, efforts were made to provide a quantitative status for the cleaning test. In this method, a metal plate (stainless steel) was homogeneously covered with a $50 \mu \mathrm{m}$ layer of epoxy paint divided into quadrangular areas of $10 \times 10 \mathrm{~mm}$ and the time necessary to remove epoxy paint from this $1 \mathrm{~cm} 2$ using the UDS at $80 \%$ power level was measured and correlated with a 1-10 ordinal scale with established intervals (Table 1). 
Am. J. Applied Sci., 7 (3): 290-300, 2010

Table 1: Intervals of time (sec) defined and attributed score

\begin{tabular}{lc}
\hline Intervals & Score \\
\hline $\mathbf{t} \leq 30$ & 10 \\
$30<\mathrm{t} \leq 60$ & 9 \\
$60<\mathrm{t} \leq 90$ & 8 \\
$90<\mathrm{t} \leq 120$ & 7 \\
$120<\mathrm{t} \leq 150$ & 6 \\
$150<\mathrm{t} \leq 180$ & 5 \\
$180<\mathrm{t} \leq 210$ & 4 \\
$210<\mathrm{t} \leq 240$ & 4 \\
$240<\mathrm{t} \leq 270$ & 3 \\
$\mathbf{t}>270$ & 2 \\
\hline
\end{tabular}

A lower time to remove the epoxy paint correlated with a higher score. To ensure as much as possible the reproducibility between each measurement in this research, a researcher experienced with the use of ultrasonic dental scalers was designed as the operator.

Cleaning test assessments: The transducers and hardware/electronics were tested using the cleaning test with the same tip type and power level of the cavitometer assessments. Thus, four equivalent repeated assessments were performed in each one of the four hardware/electronics permutations with each of the 48 Jet1 and of the 12 Jet2 UDS transducers; i.e., each transducer was measured 16 times.

Statistical analysis: Spearman test was employed to observe if there was a correlation between the scores from the cleaning test and the $\mathrm{mFM}$ values. To compare the $\mathrm{mFM}$ results obtained for the two UDS transducer types, the values were analyzed using a Student's t test $(p<0.05)$. To compare score values, the Mann Whitney $\mathrm{U}$ test $(\mathrm{p}<0.05)$ was used.

\section{RESULTS}

Acoustic emission sensor frequency band: Figure 5 shows the impedance module $(|\mathrm{Z}|)$ and the ratio $1 /(2 \pi f|Z|)$ from the acoustic sensor as a function of frequency. For frequencies below and far from the resonance, the ratio $1 /(2 \pi f|Z|)$ equals the piezoelectric ceramic element capacitance. The dashed line indicates the frequency range of interest $(5-17.5 \mathrm{kHz})$; this range is clearly in the linear region and far from the sensor resonance frequency that occurs around $72 \mathrm{kHz}$.

Power adjustment and tip type influence on mFM: Figure 6 shows the mFM dependence for the UDS power adjustment. Figure 7 shows the values of $\mathrm{mFM}$ for three different types of tips. Regarding the type of tip, the variation of the $\mathrm{mFM}$ reached $14 \%$ (type $2 \mathrm{Vs}$ type 3).

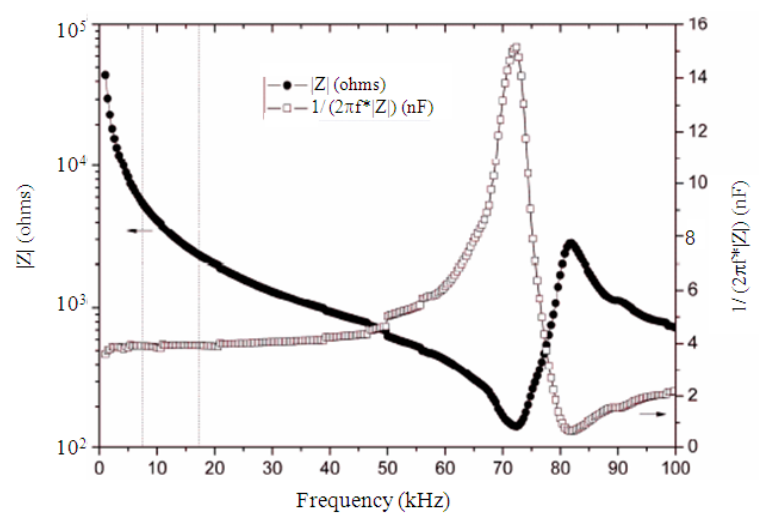

Fig. 5: Impedance spectrometry of acoustic emission sensor. The dashed lines indicate the frequency range of interest $(5-17.5 \mathrm{kHz})$. The sensor first resonance occurs around $72 \mathrm{kHz}$

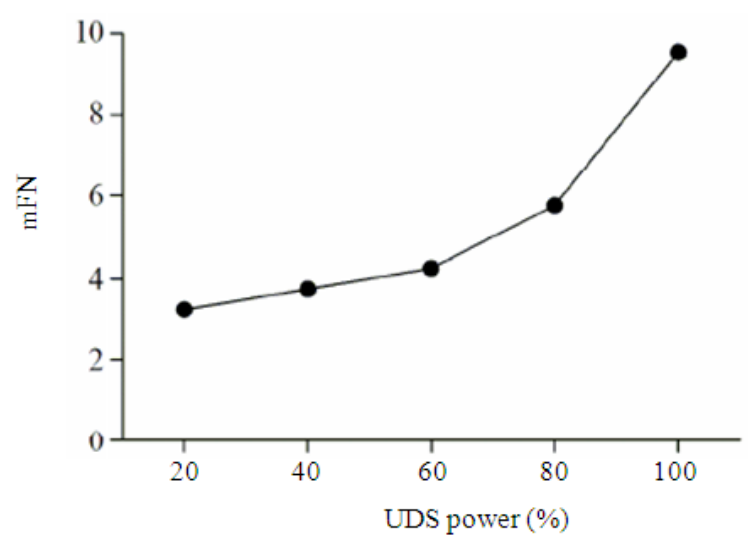

Fig. 6: The mFM dependence for the UDS dial power adjustment. The slope is constant between 20 and $60 \%$ and starts to increase after $60 \%$

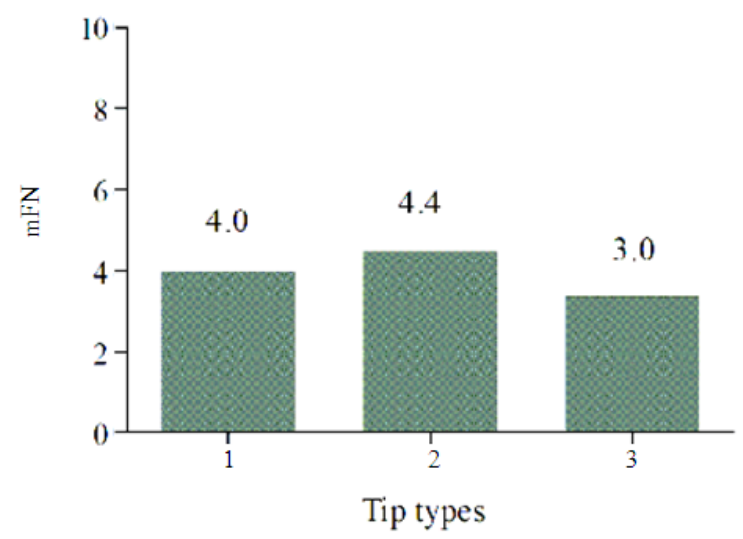

Fig. 7: Values of $\mathrm{mFM}$ for the same UDS with three different types of tips 


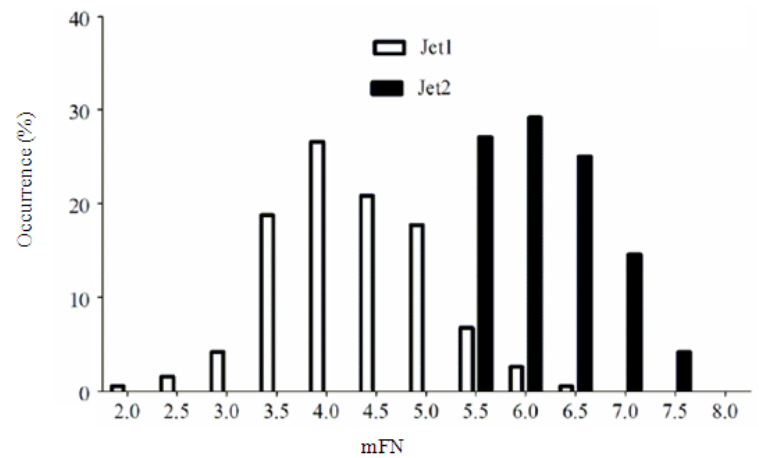

Fig. 8: Frequency distributions of $\mathrm{mFM}$ given by the developed cavitometer for Jet1 and Jet2 assessments with the developed cavitometer

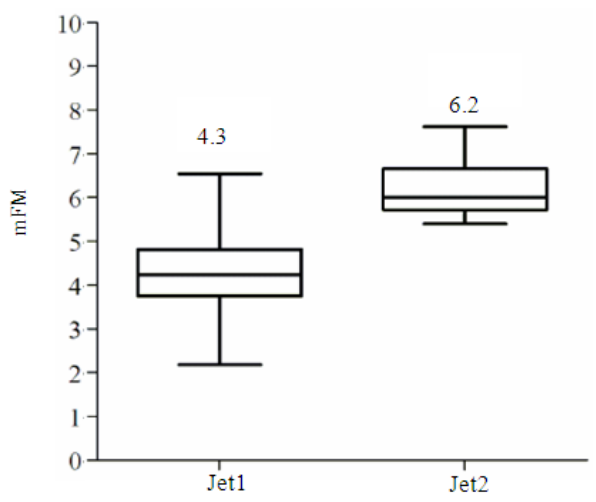

Fig. 9: Box chart of the mFM values obtained from the Jet1 and Jet2 transducers with the developed cavitometer. There is a statistical difference between the means $(\mathrm{p}<0.001)$ of Jet 1 $(\mathrm{mFM}=4.3 \pm 0.7)$ and Jet2 $(\mathrm{mFM}=6.2 \pm 0.5)$

Cavitometer assessments: Figure 8 shows the mFM values and frequency distributions obtained with the developed cavitometer for Jet1 and Jet2 and Fig. 9 shows the mFM values box chart. There is a statistical difference between the means $(p<0.001)$ of Jet1 $(\mathrm{mFM}=4.3 \pm 0.7)$ and Jet2 $(\mathrm{mFM}=6.2 \pm 0.5)$. Considering that the $\mathrm{mFM}$ is associated with the inertial cavitation and possibly with the performance, one can expect that the Jet2 transducers are superior with respect to Jet1. The reproducibility between measurements can be deducted from the observed standard deviation.

Cleaning test assessments: Figure 10 shows the frequency distributions given by the cleaning test. The results obtained with the comparative method showed a statistical difference between the mean scores of Jet1 (7.0 \pm 0.8$)$ and Jet2 $(8.7 \pm 0.4)$, signaling that Jet2 was more efficient in removing epoxy paint from a metal plate than Jet1 $(\mathrm{p}<0.0001)$.

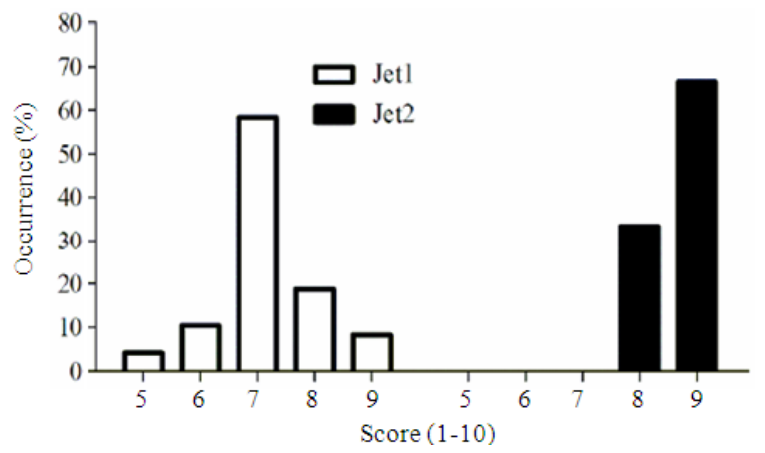

Fig. 10: Frequency distributions of scores observed with the cleaning test

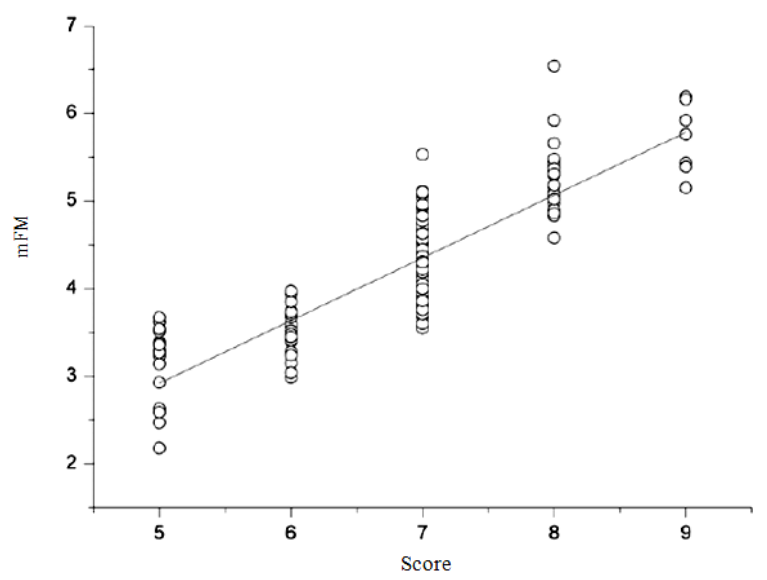

Fig. 11: Graphic illustration of the correlation observed between the measurements provided by the cavitometer and the cleaning test

Through the cleaning test and developed cavitometer, it was observed that the Jet2 transducers were superior to the Jet 1 transducers. In the case of the Jet2 transducers, all 12 units received the highest scores when analyzed with the cleaning test and the highest mFM when analyzed with the cavitometer. Similarly, for the Jet1 transducers, the lowest scores obtained were associated with minimum $\mathrm{mFM}$ values, which show that the developed cavitometer was able to detect transducers with low performance.

From Fig. 8 and 11, there are differences in the frequency distribution variance in contrast to the cleaning test. In Figure 8, when the Jet1 sample was evaluated using the cavitometer, around $40 \%$ of the sample received mFM values bellow the mean (4.3). When this same sample was evaluated by the cleaning test, approximately $15 \%$ of the scores were at the left of the mean (7.0). This observation suggests the possibility of falsepositive assessments by the operator, 
or the opposite, false-negatives by the developed cavitometer.

Correlation between cleaning test and cavitometer values: A correlation between the score values from the cleaning test and the mFM from the cavitometer was found. As the cleaning test was the reference method, it was necessary to observe the correlation between its measurements and those provided by the cavitometer. The Spearman test indicates a correlation $(\mathrm{p}<0.0001)$ when the Jet 1 sample was analyzed by the cleaning test and by cavitometer; a correlation was also noted for Jet2 $(p<0.006)$. Figure 11 shows the results of the $\mathrm{mFM}$ obtained using the cavitometer versus the score obtained with the cleaning test. The line is to guide the eyes and results from a linear interpolation. The statistical test used does not ask for a linear relation between the variables analyzed.

The point's distribution in Fig. 11 would be a cloud instead of straight lines parallel to the $\mathrm{mFM}$ axis if the scores were not discrete, which also broadens the dispersion of the $\mathrm{mFM}$ values corresponding to the score values.

\section{DISCUSSION}

Notwithstanding its wide use and the great potential of the UDS application, to the best of our knowledge, there are no standard methods to control its performance. However, great efforts have been made by researchers and manufacturers to quantitatively ensure UDS performance. Thus, the challenge of this study was to reach a practical and effective method for the quantitative evaluation of UDS. It is important to note that the equipment developed here, based on an innovative acoustic sensor, was able to migrate from a laboratory scale measurement to an industrial scale replacing a qualitative and human judgment based test, which is further experimental evidence that the development was successful.

The results from the acoustic emission sensor that considers the adjustment power of the UDS showed similar behavior when compared with that found by Liang et al. (2006) for the non-linear component of the cavitation; however, it was not verified if the UDS power adjustment employed in this study was really linear, as indicated by its dial. Nonetheless, fixing the power adjustment at a specific number was important to aid the standardization of the evaluations.

The most sensitive parameter in the $\mathrm{mFM}$ variation was the tip type, which was responsible for a variation of up to $14 \%$ among the transducers tested. This result was expected as Lea et al. (2005) had related that the geometry of the scaler tips affects the magnitude of their vibration displacement amplitude. Tip type 2 was used to carry out the comparative evaluations. In addition, we must consider that other factors of variation, such as the aqueous solution temperature, or even electrical parameters, including the voltage of the power network, may contribute to variation among measurements with the same type of tip. The mFM variation was between 5 and $7 \%$.

The cavitometer was developed to substitute the cleaning test in one industrial UDS quality control. Due to this fact, it was necessary to compare both methods to assure the cavitometer acceptance. Regarding this comparative observation, a correlation was found between the cleanness and cavitation produced by the UDSs evaluated; however, no proportion for this correlation, such as 1:1 for instance, is assumed in this research. In order to decrease possible drawbacks in comparing measurements from these two methods, two different and large samples (48 and 12 transducers) and a consistent number of assessments were employed and a researcher was instructed to execute each epoxy paint removal as reproducible as possible.

The quantitative results from the cavitometer compared with the cleaning capacity led us to the assumption that there was a convergent diagnostic for the transducers, which indicated the superiority of Jet 2 . The observation shows that Jet2 received the highest evaluations for both $\mathrm{mFM}$ and score from cavitometer and cleaning test, respectively. However, there was a significant difference between the evaluations. The developed cavitometer attributed around $40 \%$ of the Jet1 transducer values of the mMF not reaching the overall medium value of 4.3 , while the operator using the cleaning test detected a similar situation for $15 \%$ of the transducers. This difference between both analyses indicates that the cavitometer provides a more precise evaluation regarding UDS performance or even falsenegative diagnosis.

Further improvements in the equipment and methodology are being designed and further comparisons are being planned between the $\mathrm{mFM}$ obtained from acoustic emission induced by the UDS in the aqueous solution and other quantitative methods in order to strengthen the validation of the diagnostic provided by the novel cavitometer and the direct association with the UDS performance.

\section{CONCLUSION}

The developed cavitometer was shown to be adequate for the quantitative evaluation of ultrasonic dental scalers in industrial production line quality 
control using the acoustic emission produced by the cavitation induced by the tip of the transducers when immersed in aqueous solution.

\section{ACKNOWLEDGMENTS}

We would like to acknowledge the loan of a generators, piezoletric transducers and tip types (Jetsonic equipment parts) by Gnatus- Medical and Dental Equipments, Ribeirão Preto, SP, Brazil.

\section{REFERENCES}

Arabaci, T., Y. Cicek and C.F. Canakci, 2007. Sonic and ultrasonic scalers in periodontal treatment: A review. Int. J. Dental Hyg., 5: 2-12. PMID: 17250573

Atchley, A.A., L.A., Frizzell, R.E. Apfel, C.K. Holland, S. Madanshetty and R.A. Roy, 1988. Thresholds for cavitation produced in water by pulsed ultrasound. Ultrasonics, 26: 280-285. PMID: 3407017

Busslinger, A., K. Lampe, M. Beuchat and B. Lehmann, 2001. A comparative in vitro study of a magnetostrictive and a piezoelectric ultrasonic scaling instrument. J. Clin. Periodontol., 28: 642649. PMID: 11422585

Clark, S.M., 1969. The ultrasonic dental unit: A guide for the clinical application of ultrasonics in dentistry and in dental hygiene. J. Periodontol., 40: 621-629. PMID: 4901009

Drisko, C.H., 1998. Root instrumentation. Powerdriven versus manual scalers, which one? Dental Clin. North Am., 42: 229-244. PMID: 9597335

Felver, B., D.C. King, S.C. Lea, G.J. Price and A.D. Walmsley, 2009. Cavitation occurrence around ultrasonic dental scalers. Ultrason. Sonochem., 16: 692-697. PMID: 19119051

Frohly, J., S. Labouret, C. Bruneel, I. Looten-Baquet and R. Torguet, 2000. Ultrasonic cavitation monitoring by acoustic noise power measurement. J. Acoust. Soc. Am., 108: 2012-2020. PMID: 11108340

Hodnett, M. and B. Zeqiri, 1997. A strategy for the development and standardisation of measurement methods for high power/cavitating ultrasonic fields: Review of high power filed measurement techniques. Ultrason. Sonochem., 4: 273-288. PMID: 11233809

Guentsch, A. and P.M. Preshaw, 2008. The use of a linear oscillating device in periodontal treatment: A review. J. Clin. Periodontol., 35: 514-524. PMID: 18410394
Johnson, W.N. and J.R. Wilson, 1957. Application of the ultrasonic dental unit to scaling procedures. J. Periodontol., 28: 264-271. PMID: 15766378

Lea, S.C., G. Landini and A.D. Walmsley, 2002. Vibration characteristics of ultrasonic scalers assessed with scanning laser vibrometry. J. Dent., 30: 147-151. PMID: 12450721

Lea, S.C., G. Landini and A.D. Walmsley, 2003a. Displacement amplitude of ultrasonic scaler inserts. J. Clin. Periodontol., 30: 505-510. PMID: 12795788

Lea, S.C., G. Landini and A.D. Walmsley, 2003b. Ultrasonic scaler tip performance under various load conditions. J. Clin. Periodontol., 30: 876-881. PMID: 14629574

Lea, S.C., G. Landini and A.D. Walmsley, 2004. Assessing the vibrations of dental ultrasonic scalers. J. Sound Vibrat., 271: 1113-1120. doi:10.1016/j.jsv.2003.06.014

Lea, S.C., G.J. Price and A.D. Walmsley, 2005. A study to determine whether cavitation occurs around dental ultrasonic scaling instruments. Ultrason. Sonochem., 12: 233-236. PMID: 15491887

Lea, S.C., G. Landini and A.D. Walmsley, 2006. The effect of wear on ultrasonic scaler tip displacement amplitude. J. Clin. Periodontol., 33: 37-41. PMID: 16367854

Lea, S.C., B. Felver, G. Landini and A.D. Walmsley, 2009a. Three-dimensional analyses of ultrasonic scaler oscillations. J. Clin. Periodontol., 36: 44-50. PMID: 19017036

Lea, S.C., B. Felver, G. Landini and A.D. Walmsley, 2009b. Ultrasonic scaler oscillations and toothsurface defects. J. Dental Res., 88: 229-234. PMID: 19329455

Leighton, T.G., 2007. What is ultrasound? Prog. Biophys. Mol. Biol., 93: 3-83. PMID: 17045633

Liang, Z., G. Zhou, S. Lin, Y. Zhang and H. Yang, 2006. Study of low-frequency ultrasonic cavitation fields based on spectral analysis technique. Ultrasonics, 44: 115-120. PMID: 16289194

Oda, S., H. Nitta, T. Setoguchi, Y. Izumi and I. Ishikawa, 2000. Current concepts and advances in manual and power-driven instrumentation. Periodontology, 36: 45-58. PMID: 15330943

Walmsley, A.D., W.R.E. Laird and A.R. Williams, 1984. A model system to demonstrate the role of cavitational activity in ultrasonic scaling. J. Dent. Res., 63: 1162-1165. PMID: 6379010

Walmsley, A.D., W.R.E. Laird and A.R. Williams, 1986. Displacement amplitude as a measure of the acoustic output of ultrasonic scalers. Dent. Mater., 2: 97-100. PMID: 3525294 
Walmsley, A.D., S.C. Lea, G. Landini and A.J. Moses, 2008. Advances in power driven pocket/root instrumentation. J. Clin. Periodontol., 35: 22-28. PMID: 18724839

Zeqiri, B., P.N. Gélat, M. Hodnett and N.D. Lee, 2003. A novel sensor for monitoring acoustic cavitation. Part I: Concept, theory and prototype development. IEEE Trans. Ultrason. Ferroelect. Freq. Cont., 50: 1342-50. PMID: 14609074
Zitterbart, P.A., 1987. Effectiveness of ultrasonic scalers: A literature review. Gen. Dent., 35: 295297. PMID: 3327733 